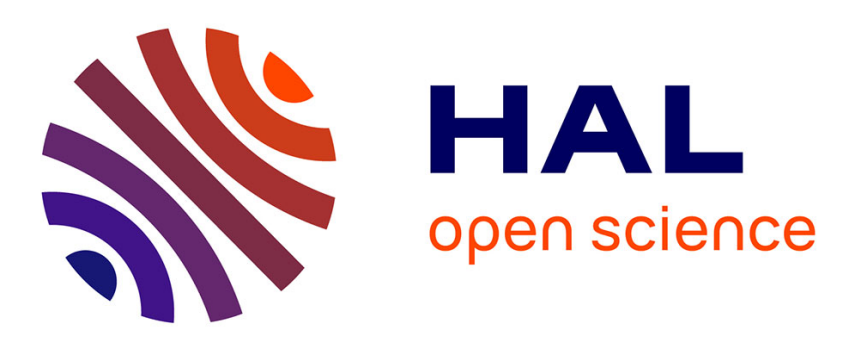

\title{
A Monte Carlo estimation of the mean residence time in cells surrounded by thin layers
}

\author{
Antoine Lejay
}

\section{To cite this version:}

Antoine Lejay. A Monte Carlo estimation of the mean residence time in cells surrounded by thin layers. Mathematics and Computers in Simulation, 2018, Tenth IMACS Seminar on Monte Carlo Methods (MCM 2015), 143C, pp.65-77. 10.1016/j.matcom.2017.05.008 . hal-01216471v6

\section{HAL Id: hal-01216471 \\ https://hal.inria.fr/hal-01216471v6}

Submitted on 11 Jun 2017

HAL is a multi-disciplinary open access archive for the deposit and dissemination of scientific research documents, whether they are published or not. The documents may come from teaching and research institutions in France or abroad, or from public or private research centers.
L'archive ouverte pluridisciplinaire HAL, est destinée au dépôt et à la diffusion de documents scientifiques de niveau recherche, publiés ou non, émanant des établissements d'enseignement et de recherche français ou étrangers, des laboratoires publics ou privés.

$$
\text { Copyright }
$$




\title{
A Monte Carlo estimation of the mean residence time in cells surrounded by thin layers
}

\author{
Antoine Lejay*
}

May 28, 2017

\begin{abstract}
We present a new Monte Carlo method to estimate the mean-residence time of a diffusive particle in a domain surrounded by a thin layer of low diffusivity. Through a homogenization technique, the layer is identified with a membrane. The simulations use a stochastic process called the snapping out Brownian motion the density of which matches suitable transmission conditions at the membrane. We provide a benchmark test which is a simplified form of a real-life problem coming from brain imaging techniques. We also provide a new algorithm to adaptively estimate the exponential rate of the tail of the distribution function of the probability to be in the domain using Monte Carlo simulations.
\end{abstract}

Keywords: semi-permeable membrane, elastic Brownian motion, snapping out Brownian motion, thin layer problem, mean-residence time, Monte Carlo methods, homogenization, first eigenvalue estimation.

\section{Introduction}

Understanding the dynamics of a diffusive process in a medium with interfaces is of great importance both for modeling and simulation. An interface could be a permeable or a semi-permeable barrier. It arises as a limit of a sharp change in the properties of the underlying material. In geophysics, the inclusion of rocks, fissures, ..., leads to such interfaces, just to cite one among the many possible domains of applications (see, e.g., [2, 13]).

We consider here a medium with a "thin layer" of low diffusivity seen in the limit as a semipermeable barrier the particle has difficulty to pass through (see [22, Chap. 13] or [19] for a proof relying on homogenization techniques, and [10, Th. 2] for a probabilistic proof in one dimension). The membrane surrounding living cells is an example of such a layer. A brain imaging technique such as diffusion Magnetic Resonance Imaging (dMRI) records the mean square displacement of particles of water [6]. Reconstructing the brain activity is done by estimating the diffusion coefficient through an inverse problem. Understanding the diffusive behavior of particles in living tissue, where thin layers are frequently present, is then essential for applying Monte Carlo methods [17].

In brain imaging, the mean residence time (MRT) in the cells is an important macroscopic parameter related to the rate of convergence toward equilibrium. It could serve as an input for simplified,

*Université de Lorraine, IECL, UMR 7502, Vandœuvre-lès-Nancy, F-54500, France;

CNRS, IECL, UMR 7502, Vandœuvre-lès-Nancy, F-54500, France;

Inria, Villers-lès-Nancy, F-54600, France

email: Antoine.Lejay@univ-lorraine.fr 
homogenized models such as double porosity models [3, 4, 16, 21]. The MRT is also commonly used in pharmacokinetics and geophysics [15, 20, 25].

We propose a way to estimate the MRT in media containing thin layers with Monte Carlo simulations using continuous processes instead of random walks as in [21]. We simulate paths of a stochastic process the density of which solves the PDE giving the concentration of the species of interest. To avoid the large computational cost induced by the layers, which forces to use a small time step around it, we replace the process by a simpler one called the snapping out Brownian motion [10]. This is equivalent to replacing the thin layer by a semi-permeable membrane. We then show how to estimate the MRT from the Monte Carlo simulation. By the ways, we improve the estimation procedure of a first eigenvalue problem proposed in [12]. Finally, we provide a one-dimensional benchmark test which shows the effectiveness of our approach unless the permeability of the membrane is too low.

\section{A Monte Carlo estimation of the mean residence time}

The concentration $C(t, x)$ of the diffusive species (water, ...) follows an equation of the type

$$
\partial_{t} C(t, x)=\nabla \cdot(D(x) \nabla C(t, x)), t>0, x \in \Omega
$$

in a box $\Omega$ of dimension $d=1,2,3$ with a scalar diffusivity and periodic boundary conditions. Equivalently, we could solve (1) on a periodic medium composed of property translated copies of $\Omega$. We consider here a simplified situation where $\Omega$ contains an interior part $\Omega_{\text {intra }}$ separated from an exterior part $\Omega_{\text {extra }}$ by a layer $\Omega_{\text {layer }}$ of constant width $\varepsilon>0$. The surface separating $\Omega_{\text {layer }}$ and $\Omega_{\text {intra }}$ (resp. $\Omega_{\text {extra }}$ ) is denoted by $\Gamma_{-}$(resp. $\Gamma_{+}$). We assume that $\Gamma_{ \pm}$are of class $\mathscr{C}^{2}$ (See Figure 1 ).

For $D_{0}, \mu>0$, the diffusivity is $D(x)=D_{0}$ for $x \in \Omega_{\text {intra }} \cup \Omega_{\text {extra }}$, while $D(x)=D_{\text {layer }}=\mu \varepsilon$ for $x \in \Omega_{\text {layer }}$.

Under the above conditions, (1) is well posed and has a unique (up to an additive constant), periodic solution for any initial condition which is bounded and measurable.

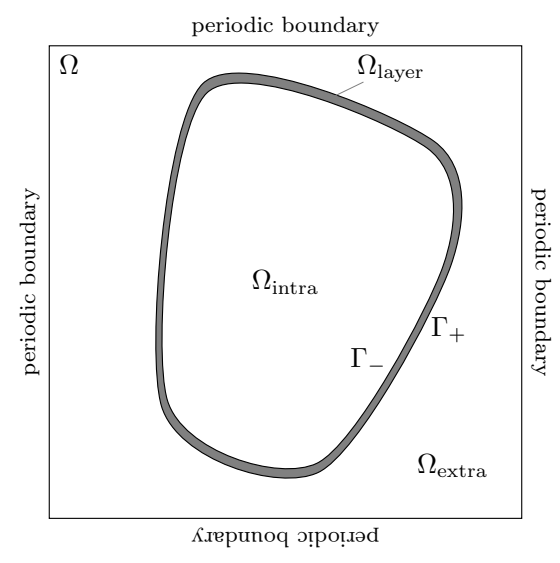

Figure 1: A box $\Omega$ of the periodic medium.

\subsection{The transmission condition}

Since $D$ is constant except on the interfaces $\Gamma_{-}$and $\Gamma_{+}$, we recast (1) into a transmission problem.

We denote by $n_{-}(x)$ (resp. $n_{+}(x)$ ) the normal derivative at a point $x \in \Gamma_{-}$(resp. $x \in \Gamma_{+}$) which is directed toward $\Omega_{\text {intra }}$ (resp. $\Omega_{\text {extra }}$ ). The concentration $C$ is of class $\mathscr{C}^{1}$ on the left and right of $\Gamma_{ \pm}$. Besides, it satisfies

$$
\partial_{t} C(t, x)=D(x) \triangle C(t, x), t>0, x \notin \Gamma_{ \pm},
$$


with

$$
\left[n_{ \pm}(x) \cdot D(x) \nabla C(t, x)\right]_{\Gamma_{ \pm}}=0 \text { and }[C(t, x)]_{\Gamma_{ \pm}}=0
$$

where at a point $x \in \Gamma_{ \pm}$

$$
[f(x)]_{\Gamma_{ \pm}}=\lim _{\delta \rightarrow 0} f\left(x+\delta n_{ \pm}(x)\right)-f\left(x-\delta n_{ \pm}(x)\right) .
$$

The condition (3) is called the transmission condition (see [8, $\$ I I I .13$, p. 224]). It specifies the continuity of the flow $n_{ \pm} \cdot D(x) \nabla C(t, x)$ across the interface $\Gamma_{ \pm}$as well as the continuity of the concentration. The variational formulation of (2)-(3) is given in Section A.2.

\subsection{The associated stochastic process}

For the diffusivity $D, \Omega, \Gamma_{ \pm}, \ldots$ above extended to $\mathbb{R}^{d}$ by periodicity, let us consider the stochastic process $X$ associated to the divergence form operator $\mathscr{L}=\nabla \cdot(D \nabla \cdot)$. This process is a strong Markov process with continuous paths as proved in [23]. It is actually constructed in an abstract way from its transition semigroup whose densities are bounded from above and from below by a Gaussian kernel. When $D$ is $\mathscr{C}^{1}$, the process $X$ is solution to the Stochastic Differential Equation $X_{t}=x+\int_{0}^{t} \sigma\left(X_{s}\right) \mathrm{d} B_{s}+\int_{0}^{t} \nabla D\left(X_{s}\right) \mathrm{d} s$, where $\sigma \sigma^{T}(x)=2 D(x)$. In our case, $\nabla D$ is not defined on $\Gamma_{ \pm}$. Therefore, no such representation holds for $X$.

The probability $p_{\text {cross }}$ that the particle moves at distance at least $R$ during a time step $\Delta t$ decreases exponentially fast with $R^{2} / \Delta t$ (this is a consequence of the Gaussian estimates on the density transition function of $X[23]$ ). For numerical simulations, we substitute to $\left\{X_{t+s}\right\}_{s \geq 0}$ a process $\left\{Z_{t+s}\right\}_{s \geq 0}$ with the same distribution until it leaves the ball of radius $R$ centered on $X_{t}$. In practice, $R=4 \sqrt{2 D_{0} \Delta t}$ is sufficient, as the probability $p_{\text {cross }}$ is of order $6 \times 10^{-5}$ as discussed in [13]. For away from the interfaces, the particle could be moved using Gaussian steps, which consists only in neglecting the probability to cross them.

This explains why, up to negligible errors, we focus on the dynamics of the process only at the vicinity of the layers.

Let $\phi$ be a $\mathscr{C}^{2}$ diffeomorphism of $\mathbb{R}^{d}$. Then $Y_{t}=\phi\left(X_{t}\right)$ is also a strong Markov process with continuous paths. Its infinitesimal generator $\mathscr{A}$ has domain $\operatorname{Dom}(\mathscr{A})=\phi(\operatorname{Dom}(\mathscr{L}))$. From standard computations, for $x \notin \Gamma_{ \pm}$and $f \in \operatorname{Dom}(\mathscr{L})$,

$$
\begin{aligned}
\mathscr{A} f(x)=\mathscr{L}(f \circ \phi)\left(\phi^{-1}(x)\right)=\frac{1}{2} D\left(\phi^{-1}(x)\right) \sum_{i, j, k=1, \ldots, d} \frac{\partial^{2} f}{\partial x_{j} \partial x_{k}}(x) \frac{\partial \phi_{k}}{\partial x_{i}}(x) \frac{\partial \phi_{j}}{\partial x_{i}}(x) & \\
& +D\left(\phi^{-1}(x)\right) \sum_{i, j=1, \ldots, d} \frac{\partial f}{\partial x_{j}}(x) \frac{\partial^{2} \phi_{j}}{\partial x_{i}^{2}}(x) .
\end{aligned}
$$

When $\phi$ is a translation or a rotation, $\mathscr{A} f(x)=\mathscr{L} f(\phi(x))$.

Recall that the layer $\Omega_{\text {layer }}$ is surrounded by two $\mathscr{C}^{2}$-interfaces $\Gamma_{-}$and $\Gamma_{+}$at distance $\varepsilon$. We use an argument similar to the one of [2] to reduce the problem to a "nearly 1-dimensional" one.

Proposition 1. At the vicinity of any point $\widehat{x}$ in $\Gamma_{-}$, there exists a $\mathscr{C}^{2}$ diffeomorphism $\phi$ such that $\phi\left(\Gamma_{-}\right)$ and $\phi\left(\Gamma_{+}\right)$are the hyperplanes orthogonal to $(1,0, \ldots, 0)$ passing through $\phi(\widehat{x})=0$ and $(\varepsilon, 0, \ldots, 0)$.

Proof. Up to compositions with a rotation and a translation, we may assume that our reference point is $\widehat{x}=0$ and $\Gamma_{-}=U \cap\left\{\left(\omega\left(x^{\prime}\right), x^{\prime}\right) \mid x^{\prime} \in \mathbb{R}^{d-1}\right\}$ for a smooth map $\omega: \mathbb{R}^{d-1} \rightarrow \mathbb{R}$ and a neighborhood $U$ of 0 . Since $\Gamma_{-}$is orientable, we choose this map so that points in $\Omega_{\text {intra }}$ have locally coordinates $\left(z, x^{\prime}\right)$ which satisfy $z \leq \omega\left(x^{\prime}\right), x^{\prime} \in \mathbb{R}^{d-1}$. Let $d: \mathbb{R}^{d} \rightarrow \mathbb{R}_{+}$be the distance between a point and $\Gamma_{-}$. At the vicinity of $\Gamma_{-}$, the distance is a well defined function with the same regularity as $\Gamma_{-}$ 
[5, Sect. 14.6, p. 354]. In addition, there exists a unique point $y$ in $\Gamma_{-}$such that $d(x)=|x-y|$. Let $n(x)=\left[\begin{array}{c}1 \\ -\nabla \omega\left(x^{\prime}\right)\end{array}\right]$ be the unit vector normal to $\Gamma_{-}$oriented toward the exterior of the cell at the point $x=\left(\omega\left(x^{\prime}\right), x^{\prime}\right)$. Write $\phi\left(d, x^{\prime}\right)=x+d n(x)$ with $x=\left(\omega\left(x^{\prime}\right), x^{\prime}\right)$ for $d \in \mathbb{R}$ and $x^{\prime} \in \mathbb{R}^{d-1}$. Thus, $\phi$ is locally a diffeomorphism and $\phi^{-1}$ maps $\Gamma_{-} \cap V$ to $\left\{\left(0, x^{\prime}\right) \mid x^{\prime} \in \mathbb{R}^{d-1}\right\} \cap U$ as well as $\Gamma_{+} \cap V$ to $\left\{\left(\varepsilon, x^{\prime}\right) \mid x^{\prime} \in \mathbb{R}^{d-1}\right\} \cap U$ for two neighborhoods $V$ and $U$ of 0 . This function $\phi$ is the diffeomorphism we are looking at.

Using this, we focus on the component which is normal to the interface $\Gamma_{ \pm}$. We are then is the same situation as the one described in [11].

Corollary 1. Using the diffeomorphism $\phi$ given above at the vicinity of a point $\widehat{x}$ of the layer, we set $X^{\phi}=\phi(X)$. The first component $\left(X^{\phi}\right)^{1}$ of $X^{\phi}$ is a diffusion process. Write $d(x)=D\left(\phi^{-1}(x, 0, \ldots, 0)\right)$ for $x \in \mathbb{R}$. The infinitesimal generator of $\left(X^{\phi}\right)^{1}$ is $\mathscr{P}=\nabla(d \nabla \cdot)$.

\subsection{The thin layer approximation}

The particles have difficulties in traversing the thin layer which is then seen as a semi-permeable barrier. Thanks to a homogenization result (see e.g. [22, Chap. 13] or [19]), our diffusion equation could be replaced by a simpler one where the layer enclosed between $\Gamma_{+}$and $\Gamma_{-}$is reduced to a single interface $\Gamma$ to which is associated a permeability $\mu$ (in $\mathrm{ms}^{-1}$ ). The condition at this single interface $\Gamma$ is

$$
\mu(C(t, x+)-C(t, x-))=D_{0} n_{+}(x) \cdot \nabla C(t, x) \text { and }\left[n_{+}(x) \cdot \nabla C(t, x)\right]_{\Gamma}=0, x \in \Gamma,
$$

where $C$ is the solution to the PDE

$$
\partial_{t} C(t, x)=D_{0} \triangle C(t, x) \text { for } x \notin \Gamma \text { and } t>0 .
$$

This approximation then simplifies theoretical or numerical computations in modeling [3, 4, 19, 24]. The variational formulation of (4)-(5), which is an instance of the Kedem-Katachalsky equation [19. Sect. 7.2], is given in Section A.3.

In dimension 1, a similar analysis can be carried out at the level of the stochastic process. Therefore, $X$ may be replaced by a simpler process $Y$, called a SNapping Out Diffusion (SNOD), living in a medium where the layers are replaced by semi-permeable barriers of vanishing width which we call membranes [10]. The coefficient $\mu$ in (4) is called the membrane permeability.

The state space of such a process is then a disjoint union of closed intervals, where each finite endpoint corresponds to one side of a membrane. The density transition function $p(t, x, y)$ of $X$ is solution to (4)-(5) in the variables $(t, x)$.

When a SNOD reaches a membrane, a local time clock drives a switching in which the particle is moved randomly on one or the other side of the membrane.

Using the Lamperti's type transform presented below in Proposition 2 to reduce its diffusivity to $1 / 2$ (the one of the Brownian motion), a SNOD living in $\mathbb{R}$ with one membrane at 0 becomes a SNapping Out Brownian motion (SNOB) [1, 10], a process which depends on a single parameter $\kappa>0$ related to $D_{0}$ and $\mu$.

A SNOB behaves like an elastic Brownian motion on $(-\infty, 0-] \cup[0+, \infty)$. When its local time at $0 \pm$ goes above an independent exponential random variable of mean $1 / \kappa$, the process is displaced with probability $1 / 2$ to either at $0+$ or $0-$. It starts afresh with the same dynamics. The behavior at 0 models a semi-permeable barrier the particle goes through after a random time in a Markovian manner.

The infinitesimal generator of the SNOB is $\mathscr{S}=\frac{1}{2} \triangle$. The domain of $\mathscr{S}$ contains as a core the functions satisfying the transmission conditions

$$
\nabla u(0+)=\nabla u(0-) \text { and } \kappa(u(0+)-u(0-))=2 \nabla u(0)
$$

and which are bounded and of class $\mathscr{C}^{2}$ elsewhere with a bounded first and second order derivatives. 
Proposition 2 ([9, Sect. 6]). Let $Y$ be a SNOD in a one-dimensional media of constant diffusivity $D_{0}$ with one membrane at 0 of permeability $\mu$. With $\psi(x)=y / \sqrt{2 D_{0}}$, the process $Y^{\psi}=\psi(Y)$ is a SNOB of parameter $\kappa=2^{3 / 2} \mu / \sqrt{D_{0}}$ when restricted to the vicinity of 0 .

When $f$ is a bounded, continuous function and $Z$ a SNOB of parameter $\kappa$, it was shown in [10] that

$$
\mathbb{E}_{x}\left[f\left(Z_{t}\right)\right]=\mathbb{E}_{x}\left[\frac{1+e^{-\kappa L_{t}^{0}(B)}}{2} f\left(\left|B_{t}\right|\right)\right]+\mathbb{E}_{x}\left[\frac{1-e^{-\kappa L_{t}^{0}(B)}}{2} f\left(-\left|B_{t}\right|\right)\right] \text { for } x \geq 0,
$$

where $B$ is a Brownian motion and $L_{t}^{0}(B)$ its symmetric local time at 0 .

Using the representation (6) of the semigroup and some explicit formula for the simulation of the local time [14], a simulation algorithm could be given for the SNOB which is presented in [9, 10].

The tangential components behave like a Brownian motion and are then easily simulated.

\subsection{A Monte Carlo estimator of the mean residence time}

The mean residence time (MRT) is an important macroscopic quantity that could be used to construct two-scale models such as in [3].

In the box $\Omega$, the operator $\check{\mathscr{L}}=\nabla(D \nabla \cdot)$ with domain $\operatorname{Dom}(\check{\mathscr{L}})=\left\{f \in \check{\mathrm{L}}^{2}(\Omega) \mid \check{\mathscr{L}} f \in \check{\mathrm{L}}^{2}(\Omega)\right\}$ is self-adjoint with a compact resolvent (here, $\check{\mathrm{L}}^{2}(\Omega)$ is the space of square integrable $\Omega$-periodic functions, see Section A.2). In particular, it admits a spectral decomposition $0 \leq \lambda_{0}<\lambda_{1}<\cdots$ with eigenfunctions $\eta_{0}, \eta_{1}, \ldots$ chosen orthonormal for the $\breve{\mathrm{L}}^{2}(\Omega)$ scalar product (regarding the sign of the eigenvalues, we use the convention $\mathscr{L} \eta_{k}=-\lambda_{k} \eta_{k}$ ). The first eigenvalue is $\lambda_{0}=0$ and its associated eigenfunction is $\eta_{0}(x)=1 / \sqrt{V_{\mathrm{per}}}$, where $V_{\mathrm{per}}$ is the volume of the box $\Omega$.

Proposition 3 ([[16, Eq. (6)] or [9, § 2.1]). The mean residence time $\tau$ in the cell satisfies the approximation

$$
\frac{1}{\tau} \approx \frac{V_{\text {intra }}}{V_{\text {per }}} \lambda_{1},
$$

where $V_{\text {intra }}$ is the volume of interior part of the cell.

Determining the first positive eigenvalue $\lambda_{1}$ is then crucial for estimating the MRT. We explain now its relationship with the process $\check{X}$ defined as the projection of the original process $X$ on the periodic box $\Omega$.

The density transition function $p$ with respect to the Lebesgue measure of $\check{X}$ admits the spectral expansion

$$
p(t, x, y)=\sum_{k=0}^{+\infty} e^{-\lambda_{k} t} \eta_{k}(x) \eta_{k}(y)
$$

Since $\eta_{0}(x)$ is constant and for a fixed point $x \in \Omega$,

$$
P(t)=\int_{\Omega_{\text {intra }}} p(t, x, y) \mathrm{d} y=\frac{V_{\text {intra }}}{V_{\text {per }}}+\Phi(x) e^{-\lambda_{1} t}+\mathrm{o}\left(\exp \left(-\lambda_{1} t\right)\right)
$$

with $\Phi(x)=\sum_{k \text { s.t. } \lambda_{k}=\lambda_{1}} \eta_{k}(x) \int_{\Omega_{\text {intra }}} \eta_{k}(y) \mathrm{d} y$, since the multiplicity of $\lambda_{1}$ is not necessarily 1 .

As $t$ increases, $P(t)$ converges to the ratio $P_{\infty}=V_{\text {intra }} / V_{\text {per }}$. Thus, $\lambda_{1}$ is actually the exponential rate of convergence toward equilibrium.

On the other hand, $P(t)=\mathbb{P}_{x}\left[\check{X}_{t} \in \Omega_{\text {intra }}\right]$. By simulating a large number $N$ of paths of the stochastic process $\check{X}$, we estimate $P(t)$ at some given times $\left\{t_{k}\right\}_{k=1, \ldots, M}$ using the empirical estimators

$$
P_{N}\left(t_{k}\right)=\frac{1}{N} \sum_{i=1}^{N} \mathbb{1}_{\check{X}_{t_{k}}^{(i)} \in \Omega_{\text {intra }}},
$$

where $\check{X}^{(1)}, \ldots, \check{X}^{(N)}$ are $N$ independent samples of the paths $\check{X}$. 
Remark 1. For practical computation, we use the SNOD approximation $\check{Y}$ of $\check{X}$ so that any thin interface is reduced to a single membrane. A similar analysis, carried out on its infinitesimal generator and eigensystem, shows that $\mathbb{P}_{x}\left[\check{Y}_{t} \in \Omega_{\text {intra }}\right] \approx P(t)$ for $t \geq 0$.

From the knowledge of $\left\{P_{N}\left(t_{k}\right)\right\}_{k=1, \ldots, M}$, we estimate $\lambda_{1}$ through a linear regression on $\left\{\log \left(P_{N}\left(t_{k}\right)-\right.\right.$ $\left.\left.P_{\infty}\right)\right\}_{k=1, \ldots, M}$. However, the first and last times $t_{1}$ and $t_{M}$ shall be carefully chosen. For this, we use the procedure presented in Section 3 .

\section{A procedure to estimate some exponential rate from Monte Carlo measurement}

Let $P(t)$ be the probability of an event, which decreases to $P_{\infty}$ with the time $t$ at some exponential rate $\lambda$. This is typical for first eigenvalues problems, such as (8) and the estimation of survival probability of a Brownian motion killed when it exits from a domain [12].

More precisely, we are willing to estimate $\lambda$ under the assumption that

$$
P(t)=P_{\infty}+c \exp (-\lambda t)+r(t) \text { with } r(t)=\mathrm{o}_{t \rightarrow \infty}(\exp (-\lambda t)),
$$

where $P_{\infty} \geq 0$ is known, yet $c>0, \lambda>0$ and $r$ are unknown. In particular, we ignore how fast $r(t) \exp (-\lambda t)$ decreases to 0 .

At different times $0<t_{1}<\cdots<t_{M}$, this quantity $P\left(t_{i}\right)$ is estimated by $P_{N}\left(t_{i}\right)$ from a Monte Carlo estimation with $N$ independent samples. A Gaussian confidence interval allows one to assert that with a probability $\alpha$,

$$
\left|P(t)-P_{N}(t)\right| \leq c_{\alpha} \frac{\sqrt{P(t)(1-P(t))}}{\sqrt{N}} \text { for } N \text { large enough and } t>0,
$$

with, e.g., $c_{\alpha}=2.57$ for $\alpha=99 \%$.

From (10),

$$
\log \left(P_{N}(t)-P_{\infty}\right)=\log c-\lambda t+\xi(t)
$$

with

$$
\xi(t)=\log \left(1+e^{\lambda t} r(t)+e^{\lambda t}\left(P_{N}(t)-P(t)\right)\right) \approx e^{\lambda t} r(t)+e^{\lambda t}\left(P_{N}(t)-P(t)\right) .
$$

The difficulty is then to find a time interval $\left[t_{-}, t_{+}\right]$so that $\xi(t)$ is small in front of $\log c$ and $\lambda t$, so that a linear regression could be used.

When $t_{-}$and $t_{+}$are fixed, $c_{\text {est }}$ and $\lambda_{\text {est }}$ are the values which minimize

$$
\mathrm{SQ}\left(t_{-}, t_{+} ; c, \lambda\right)=\sum_{\substack{i=1, \ldots, M \\ t_{-} \leq t_{i} \leq t_{+}}}\left(\log c-\lambda t_{i}-\log \left(P_{N}\left(t_{i}\right)-P_{\infty}\right)\right)^{2}=\sum_{\substack{i=1, \ldots, M \\ t_{-} \leq t_{i} \leq t_{+}}} \xi\left(t_{i}\right)^{2}
$$

The residual sum of squares is

$$
\operatorname{RSS}\left(t_{-}, t_{+}\right)=\mathrm{SQ}\left(t_{-}, t_{+} ; c_{\text {est }}, \lambda_{\text {est }}\right)=\min _{c, \lambda} \mathrm{SQ}\left(t_{-}, t_{+} ; c, \lambda\right) .
$$

Several criteria have been proposed in [12] to find the best time interval $\left[t_{-}, t_{+}\right]$for estimating $\lambda$ through a least squares procedure. Yet they partially rely on a graphical procedure, which we want to avoid.

The Monte Carlo error can be neglected unless $c_{\alpha} \sqrt{P(t)(1-P(t))} / \sqrt{N}$, the magnitude of $P_{N}(t)-$ $P(t)$, has the same order as $P(t)-P_{\infty} \approx c \exp (-\lambda t)$. Hence, it is useless to consider times $t$ at which

$$
c_{\alpha} \frac{\sqrt{P_{N}(t)\left(1-P_{N}(t)\right)}}{\sqrt{N}} \geq \beta\left(P_{N}(t)-P_{\infty}\right) \text { with say } \beta=\frac{1}{10} .
$$


This criterion fixes a limit for the maximum sampling time $t_{M}$ to consider. After this time, the estimations are no more relevant as being "flooded" by the Monte Carlo error.

Getting rid of $r(t)$ is more cumbersome, as this function is generally unknown.

In the report [9], we have proposed a new methodology in which $t_{-}$and $t_{+}$are determined adaptively using the Akaike Information Criteria (AIC) [18, Example 13.6, p. 179]. This approach is suitable as the number of values of $P_{N}\left(t_{i}\right)$ to use varies with $t_{-}$and $t_{+}$.

Since there are two parameters ( $c$ and $\lambda$ ) to estimate, we use the customary simplified formula for the AIC

$$
\begin{aligned}
\operatorname{AIC}\left(t_{-}, t_{+}\right) & =n \log \left(\operatorname{RSS}\left(t_{-}, t_{+}\right)\right)-n \log n+4, \\
\text { with } n & =\#\left\{t_{-} \leq t_{i} \leq t_{+} ; i=1, \ldots, M\right\} .
\end{aligned}
$$

The procedure is: We draw randomly some pairs $\left(t_{-}, t_{+}\right)$with $0 \leq t_{-}<t_{+} \leq t_{M}$. We select the one for which $\operatorname{AIC}\left(t_{-}, t_{+}\right)$is minimized.
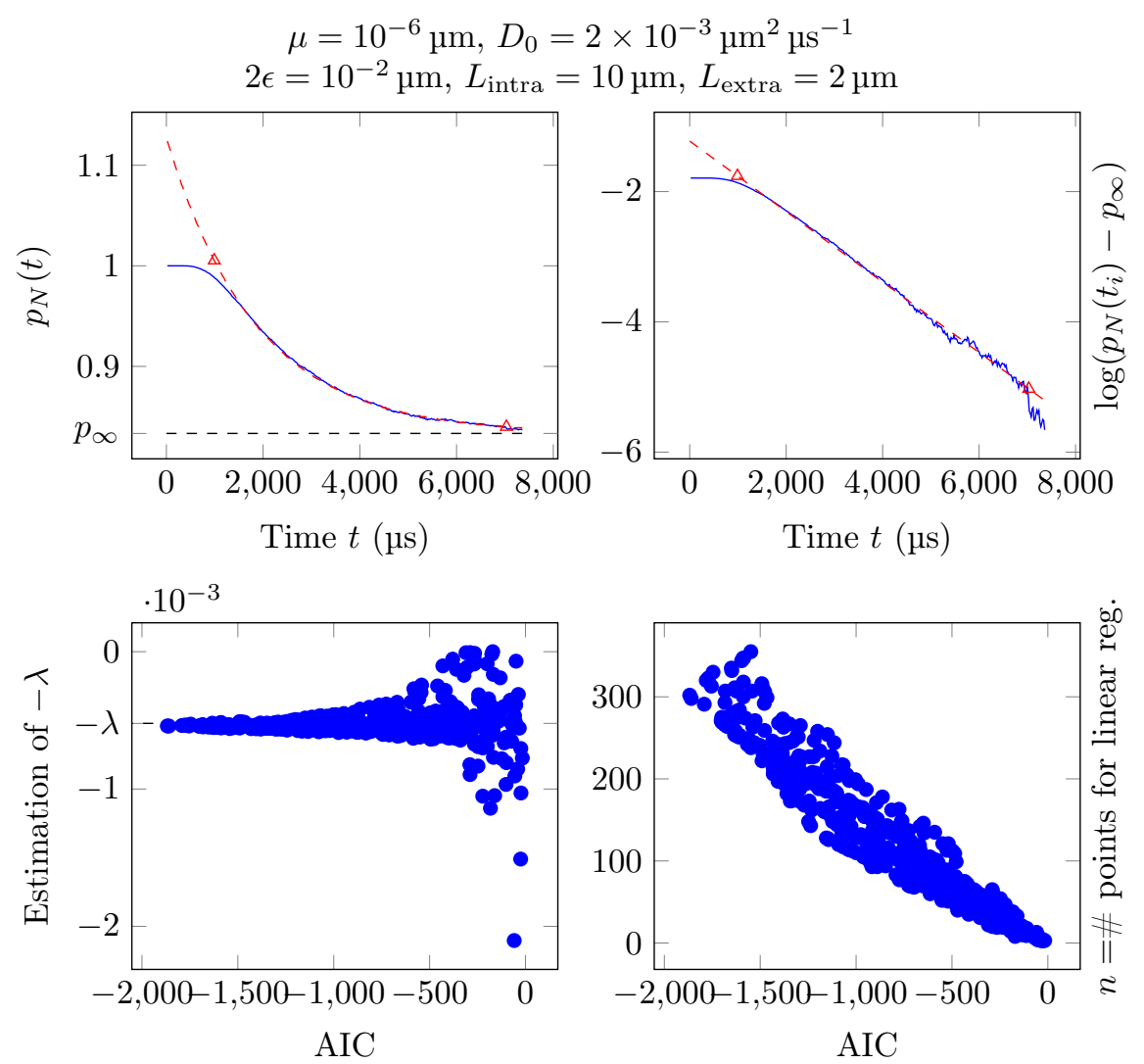

Figure 2: Estimation of $\lambda$ using a least square procedure. The curve in red represents the estimated function $c \exp (-\lambda t)$ from the time interval $\left[t_{-}, t_{+}\right]$delimited by the triangle. The two figures at the bottoms show that the values which are close to the minimum of AIC provide pretty close estimated values of $\lambda$ and use a lot of sample times. We have used $N=100000$ paths and $t_{i+1}-t_{i}=20 \mu$ s with the parameters of Table 1 .

This procedure, whose result is illustrated in Figure 2, no longer relies on a graphical interpretation as in [12].

\section{A computational benchmark test in dimension one}

We test our simulation scheme by estimating a MRT, which we compare to the numerical, deterministic estimation of an eigenvalue problem through (7). For this, we place ourselves in a one-dimensional 


\begin{tabular}{lllc}
\hline Name & Quantity & From & To \\
\hline$D_{0}$ & diffusivity in $\Omega_{\text {intra }} \cup \Omega_{\text {extra }}$ & $2 \times 10^{-3} \mu \mathrm{m}^{2} \mu \mathrm{s}^{-1}$ & $3 \times 10^{-3} \mu \mathrm{m}^{2} \mu \mathrm{s}^{-1}$ \\
$\mu \varepsilon$ & diffusivity in $\Omega_{\text {layer }}$ & $10^{-8} \mu \mathrm{m}^{2} \mu \mathrm{s}^{-1}$ & $10^{-6} \mu \mathrm{m}^{2} \mu \mathrm{s}^{-1}$ \\
$\varepsilon$ & layer width & & $0.01 \mu \mathrm{m}$ \\
$\mu=\frac{D_{\text {layer }}}{\varepsilon}$ & membrane's permeability & $10^{-6} \mu \mathrm{m} \mu \mathrm{s}^{-1}$ & $10^{-4} \mu \mathrm{m} \mu \mathrm{s}^{-1}$ \\
$V_{\text {intra }}$ & intracellular size & $5 \mu \mathrm{m}$ & $20 \mu \mathrm{m}$ \\
$V_{\text {extra }}$ & extracellular size & & $\approx V_{\text {intra }} / 10$ \\
$V_{\text {per }}$ & medium's period & & $V_{\text {intra }}+V_{\text {extra }}$ \\
$T$ & max. observations' time & $20000 \mu \mathrm{s}$ & $50000 \mu \mathrm{s}$ \\
\hline
\end{tabular}

Table 1: A realistic range of parameters.

medium, which allows explicit computations of the first positive eigenvalue $\lambda=\lambda_{1}$. We consider a cell $\Omega_{\text {intra }}$ surrounded by two thin layers at positions $y_{1}$ and $y_{2}$ in a box $\Omega$. The set of points in the layers is $\Omega_{\text {layer. }}$. We set $\Omega_{\text {extra }}=\Omega \backslash\left(\Omega_{\text {intra }} \cup \Omega_{\text {layer }}\right)$. The diffusivity is $D(x)=D_{0}$, for $x \in \Omega_{\text {intra }} \cup \Omega_{\text {extra. }}$. In each layers of width $\varepsilon$, the diffusivity is $D(x)=\mu \varepsilon$.

Using the thin layer approximation, $\Omega_{\text {layer }}$ is just reduced to just two points at the positions of the membranes replacing the layers.

Still using this approximation, we consider the process $\check{Y}$ living in the box with two membranes at $y_{1}$ and $y_{2}$, and diffusivity $D_{0}$. This process is obtained by projecting a process $Y$ living in the periodic medium. The process $Z=\check{Y} / \sqrt{2 D_{0}}$ has diffusivity $1 / 2$ outside and inside the cell. Around each membrane, $\check{Y}$ acts ${ }^{1}$ like a SNOB with parameter $\kappa=2^{3 / 2} \mu / \sqrt{D_{0}}$ (Proposition 2). We simulate $\check{Y}$ with the algorithm given in [10] to deal locally with each membrane. The impact of this choice is studied in Section B,

According to (7), the MRT is then approximated by $\tau \approx \lambda^{-1} V_{\text {per }} / V_{\text {intra }}$.

We use a realistic range of parameters given in Table 1 . We perform $N$ independent simulations. With (9), we compute empirical estimations $P_{N}\left(t_{k}\right)$ of $P\left(t_{k}\right)$ given by (8) at given times $0<t_{1}<\cdots<$ $t_{M} \leq T$. We then use the procedure of Section 3 to estimate $\lambda$.

Being the first non-zero eigenvalue of $\mathscr{L}, \lambda$ could also be computed deterministically as an eigenvalue of a Sturm-Liouville problem. Numerically, it is easily checked that the eigenvalues with the thin layer approximation are close to the ones of the original problem [9]. The process is simulated only using the thin layer approximation, which leads to faster computations. Without the thin layer approximation, the simulations could be performed using the algorithms given in [13]. Yet the thinness of the layer forces to use a very small time step, burdening the computational cost.

Numerical results are given in Figure 3. Unless $\mu$ is too small, the Monte Carlo results are in good agreements with the deterministic approach. A small value of $\mu$ means that the particles have difficulties to pass through the semi-permeable barrier. This problem is related to a rare events simulation problem. This shall be subject to future investigations.

\section{A The thin layer problem: variational formulation}

\section{A.1 Notations}

We denote by $\check{L}^{2}(\Omega)$ be the space of $\Omega$-periodic functions which are square-integrable, that is

$$
\|f\|_{\check{\mathrm{L}}^{2}(\Omega)}^{2}=\langle f, f\rangle<+\infty \text { with }\langle f, g\rangle=\int_{\Omega} f(x) g(x) \mathrm{d} x .
$$

\footnotetext{
${ }^{1}$ That is, by neglecting the excursions which are so large that $\breve{Y}$ crosses the other interface during the small time step.
} 


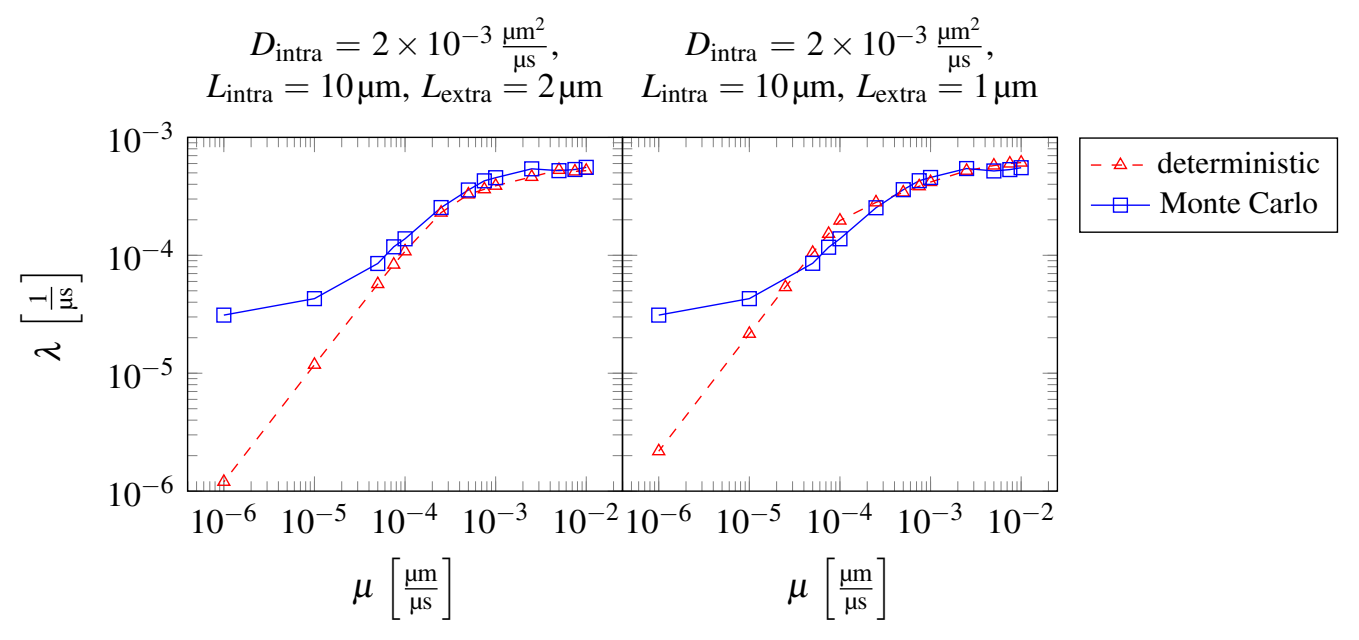

Figure 3: Estimation of $\lambda$ by a deterministic method and a Monte Carlo one with $N=100000$ particles.

For an integrable $\Omega$-periodic function $f$, we write

$$
f_{\Omega}=\frac{1}{|\Omega|} \int_{\Omega} f(x) \mathrm{d} x
$$

where $|\Omega|$ is the volume of $\Omega$.

The Sobolev space $\check{H}^{1}(\Omega)$ is the completion of $\Omega$-periodic functions of class $\mathscr{C}^{1}(\Omega)$ with respect to the norm $\|f\|_{\check{\mathrm{H}}^{1}(\Omega)}$ defined by

$$
\|f\|_{\check{\mathrm{H}}^{1}(\Omega)}^{2}=\int_{\Omega}|f(x)|^{2} \mathrm{~d} x+\int_{\Omega}|\nabla f(x)|^{2} \mathrm{~d} x .
$$

We then define

$$
\check{\mathrm{V}}=\left\{f \in \check{\mathrm{H}}^{1}(\Omega) \mid f_{\Omega}=0\right\} .
$$

As $\Omega$ is compact and owing to the periodic boundary conditions, $\check{\mathrm{H}}^{1}(\Omega)$ is compactly embedded in $\check{\mathrm{L}}^{2}(\Omega)$, and so is $\check{\mathrm{V}}$. This result is an instance of the Rellich-Kondrachev theorem.

\section{A.2 Variational formulation for the initial diffusion problem}

We consider the bilinear form

$$
E(f, g)=\int_{\Omega}\langle D(x) \nabla f(x), \nabla g(x)\rangle \mathrm{d} x, \forall(f, g) \in \check{\mathrm{H}}^{1}(\Omega)
$$

where $D$ is the diffusivity defined in Section 2 in the medium with layer of width $\varepsilon$.

From classical results, the Poincaré inequality holds for each $\varepsilon>0$ : there exists a constant $C_{\varepsilon}$ such that

$$
\int_{\Omega}\left(f(x)-f_{\Omega}\right)^{2} \mathrm{~d} x \leq C_{\varepsilon} \int_{\Omega}|\nabla f(x)|^{2} \mathrm{~d} x, \forall f \in \check{\mathrm{H}}^{1}(\Omega) .
$$

The proof of the next proposition proceeds from standard results since $E$ is bilinear, symmetric and coercive thanks to the Poincaré inequality (See e.g. [26, Chap. 22])

Proposition 4. There exists a self-adjoint operator $\check{\mathscr{L}}$ with domain $\operatorname{Dom}(\check{\mathscr{L}}) \subset \check{\mathrm{V}}$ such that

$$
E(f, g)=-\langle\check{\mathscr{L}} f, g\rangle, \forall(f, g) \in \operatorname{Dom}(\check{\mathscr{L}}) \times \check{\mathrm{V}} .
$$


Besides, for any $\lambda \geq 0, \lambda-\check{\mathscr{L}}$ is invertible and $(\lambda-\check{\mathscr{L}})^{-1}: \check{L}^{2}(\Omega) \rightarrow \operatorname{Dom}(\check{\mathscr{L}})$ is a bounded operator which is compact from $\check{\mathrm{L}}^{2}(\Omega)$ to $\check{\mathrm{L}}^{2}(\Omega)$. In particular, $\check{\mathscr{L}}$ has an eigensystem $\left\{\lambda_{k}, \eta_{k}\right\}_{k \geq 0}$ such that $\check{\mathscr{L}} \eta_{k}=-\lambda_{k} \eta_{k},\left\{\lambda_{k}\right\}_{k \geq 0}$ has no accumulation point and $\left\{\eta_{k}\right\}_{k \geq 0}$ is an orthonormal basis of $\check{\mathrm{L}}^{2}(\Omega)$.

Results on the local regularity of the solution through the interfaces $\Gamma_{+}$and $\Gamma_{-}$may be found $e . g$., in [7].

\section{A.3 Variational formulation for the thin layer approximation}

With the thin layer approximation, we define by $\Gamma=\Gamma_{+}=\Gamma_{-}$, the layer of zero width which splits $\Omega$ to $\Omega_{\text {extra }}$ and $\Omega_{\text {intra }}$.

We define

$$
\check{\mathrm{W}}=\left\{f \in \check{\mathrm{H}}^{1}(\Omega \backslash \Gamma) \mid f \text { is } \Omega \text {-periodic }, f_{\Omega}=0\right\} .
$$

Applying the Rellich-Kondrachev inequality in both $\Omega_{\text {extra }}$ and $\Omega_{\text {intra }}$ proves that $\check{\mathrm{W}}$ is compactly embedded into $\check{L}^{2}(\Omega)$.

Let us recall the trace theorem: the application $\gamma_{+}: f \in \mathscr{C}\left(\Omega_{\text {extra }}\right) \rightarrow \mathscr{C}(\Gamma)$ may be extended continuously from $\mathrm{H}^{1}\left(\Omega_{\text {extra }}\right)$ to $\mathrm{L}^{1 / 2}(\Gamma)$, the functions $g: \Gamma \rightarrow \mathbb{R}$ such that $\int_{\Gamma} g(x)^{2} \mathrm{~d} \sigma$ is finite. The trace $\gamma_{-}$on $\Gamma_{-}$is defined similarly.

Hence, for $f \in \check{\mathrm{W}},[f]_{\Gamma}=\gamma_{+} f-\gamma_{-} f$ is well defined as a function in $\mathrm{L}^{1 / 2}(\Gamma)$. Therefore $\int_{\Gamma}[f]_{\Gamma}^{2} \mathrm{~d} \sigma<$ $+\infty$, where $\sigma$ is the measure on the surface $\Gamma$.

Let us define the bilinear form

$$
S(f, g)=\int_{\Omega}\left\langle D_{0} \nabla f(x), \nabla g(x)\right\rangle \mathrm{d} x+\mu \int_{\Gamma}[f]_{\Gamma} \cdot[g]_{\Gamma} \mathrm{d} \sigma, \forall f, g \in \check{\mathrm{W}} .
$$

We now prove an inequality of Poincaré type.

Lemma 1. There exists a constant $C$ such that

$$
\|f\|_{\check{\mathrm{L}}^{2}(\Omega)}^{2} \leq C S(f, f), \forall f \in \check{\mathrm{W}}
$$

Proof. Assume that (11) is not true, meaning that there exists a sequence $\left\{f_{n}\right\}$ such that $f_{n} \in \check{\mathrm{L}}^{2}(\Omega)$ with $\left\|f_{n}\right\|_{\check{\mathrm{L}}^{2}(\Omega)}=1$ and $1 \geq C_{n} S\left(f_{n}, f_{n}\right)$ with $C_{n} \underset{n \rightarrow \infty}{\longrightarrow}+\infty$.

Applying the Poincaré inequality to both $\left(f_{n}\right)_{\mid \Omega_{\text {extra }}}$ and $\left(f_{n}\right)_{\mid \Omega_{\text {intra }}}$ implies that $f_{n}$ converges strongly to $f$ that takes a constant value $f_{+}$(resp. $f_{-}$) on $\Omega_{\text {extra }}$ (resp. $\Omega_{\text {intra }}$ ). Yet $\int_{\Gamma}\left[f_{n}\right]_{\Gamma}^{2} \mathrm{~d} \sigma$ converges to 0 . By continuity of the traces $\gamma_{+}$and $\gamma_{-}, f_{+}=f_{-}$. Since $\left(f_{n}\right)_{\Omega}=0, f_{\Omega}=0$ and thus $f=0$.

From Lemma 1 and standard results (See e.g. [26, Chap. 22]), we get the following proposition which is similar to Proposition 4.

Proposition 5. There exists a self-adjoint operator $\mathscr{S}$ with domain $\operatorname{Dom}(\mathscr{S}) \subset \mathrm{W}$ such that

$$
S(f, g)=-\langle\mathscr{S} f, g\rangle, \forall(f, g) \in \operatorname{Dom}(\mathscr{S}) \times \check{\mathrm{W}} .
$$

Besides, for any $\lambda \geq 0, \lambda-\mathscr{S}$ is invertible and $(\lambda-\mathscr{S})^{-1}: \check{\mathrm{L}}^{2}(\Omega) \rightarrow \operatorname{Dom}(\mathscr{S})$ is a bounded operator which is compact from $\breve{\mathrm{L}}^{2}(\Omega)$ to $\breve{\mathrm{L}}^{2}(\Omega)$. In particular, $\mathscr{S}$ has an eigensystem $\left\{\lambda_{k}, \eta_{k}\right\}_{k \geq 0}$ such that $\mathscr{S} \eta_{k}=-\lambda_{k} \eta_{k},\left\{\lambda_{k}\right\}_{k \geq 0}$ has no accumulation point and $\left\{\eta_{k}\right\}_{k \geq 0}$ is an orthonormal basis of $\check{\mathrm{L}}^{2}(\Omega)$.

We consider now $\Omega$-periodic functions $f, u$ and $v$ which are respectively of class $\mathscr{C}, \mathscr{C}^{1}$ and $\mathscr{C}^{2}$ on $\Omega_{\text {extra }}$ and on $\Omega_{\text {intra }}$ (assuming now that the width of the layer is $\varepsilon=0$ ) such that $D_{0} \triangle u=f$ on $\Omega_{\text {extra }} \cup \Omega_{\text {intra }}$. 
Assume that for any such $v$ as above, $S(u, v)=-\langle f, v\rangle$, that is

$$
\int_{\Omega} f(x) v(x) \mathrm{d} x=D_{0} \int_{\Omega} \nabla u(x) \cdot \nabla v(x) \mathrm{d} x+\mu \int_{\Gamma}[u]_{\Gamma} \times[v]_{\Gamma} \mathrm{d} \sigma .
$$

Using an integration by parts on $\Omega_{\text {extra }}$ and $\Omega_{\text {intra }}$, since $n_{+}=-n_{-}$,

$$
D_{0} \int_{\Gamma_{+}} \frac{\partial u}{\partial n_{+}}(x) v(x) \mathrm{d} \sigma(x)-D_{0} \int_{\Gamma_{-}} \frac{\partial u}{\partial n_{+}}(x) v(x) \mathrm{d} \sigma(x)=\mu \int_{\Gamma}[u]_{\Gamma} \times[v]_{\Gamma} \mathrm{d} \sigma,
$$

where $\Gamma_{+}$(resp. $\Gamma_{-}$) denotes the sides of $\Gamma$ oriented toward $\Omega_{\text {extra }}$ (resp. $\Omega_{\text {intra }}$ ). Taking $v$ continuous around $\Gamma$ implies that $\left[\frac{\partial u}{\partial n}\right]_{\Gamma}=0$. Taking $v=1$ or $\Gamma_{+}$and $v=0$ on $\Gamma_{-}$implies that

$$
\left.D_{0} \frac{\partial u}{\partial n_{+}}\right|_{\Gamma_{+}}(x)=\left.D_{0} \frac{\partial u}{\partial n_{+}}\right|_{\Gamma_{-}}(x)=\mu[v]_{\Gamma}(x), x \in \Gamma .
$$

This proves that if $u$ is a classical solution to the variational problem $S(u, v)=-\langle f, v\rangle$ for any $v \in \check{\mathrm{W}}$, then $u$ satisfies (12) on $\Gamma$ and thus $\mathscr{S} u=f$. Conversely, if $u$ satisfies (12) on $\Gamma$ as well as $D_{0} \triangle u=f$, then $u$ is solution to the variational problem $S(u, v)=-\langle f, v\rangle$ for any $v \in \check{\mathrm{W}}$.

\section{B Exact and approximate simulation}

Let $T$ be a time horizon and $\Delta t$ a time step so that $\Delta t=T / n$ for some integer $n$. Our estimation of the MRT relies on the simulation of the successive positions $\left\{X_{k \Delta t}\right\}_{k=0, \ldots, n}$ of the stochastic process in the medium with the membranes.

We consider the particle living in the one-dimensional periodic media of Section 4 , in the periodic box $\Omega$ of length $2\left(\alpha+\alpha^{\prime}\right)$, there are two membranes at distance $2 \alpha$ enclosing an inner cell $\Omega_{\text {intra }}$. The diffusivity is $D_{0}$ both in the inner and the outer cell.

Let $B$ be a Brownian motion. We set $\beta_{k}=\left(B_{t}\right)_{k \Delta t \leq t \leq(k+1) \Delta t}$. We also define for each $k \geq 0$, $\xi_{k}=\left\{\xi_{k, i}\right\}_{i>0}$, an array of independent uniform random variables that are used to decide whether or not the particle crosses the membrane. Thanks to the Markov property, the successive positions $X_{k \Delta t}$ are computed iteratively by $X_{(k+1) \Delta t}=\mathfrak{p}\left(X_{k \Delta t}+\Psi\left(X_{k \Delta t}, \beta_{k}, \xi_{k}\right)\right)$, where $\mathfrak{p}$ denotes the projection on the box $\Omega$ and $\Psi$ is an abstract map.

Around each membrane at $\pm \alpha$, we define an interface layer by $[ \pm \alpha-\delta, \pm \alpha+\delta]$ with $\delta=$ $4 \sqrt{2 D_{0} \Delta t}$.

A numerical approximation $\left\{Z_{k}\right\}_{k=0, \ldots, n}$ of $\left\{X_{k}\right\}_{k=0, \ldots, n}$ is computed iteratively $Z_{(k+1) \Delta t}=\mathfrak{p}\left(Z_{k \Delta t}+\right.$ $\left.\Xi\left(Z_{k \Delta t}, \beta_{k}, \xi_{k}\right)\right)$ using an approximation $\Xi$ of $\Theta$. Two rules are used to move the particle (See Figure 4). When $Z_{k \Delta t}$ does not belongs to an interface layer, we use a Gaussian step $Z_{(k+1) \Delta t}=$ $Z_{k \Delta t}+\sqrt{2 D_{0}}\left(B_{(k+1) \Delta t}-B_{k \Delta t}\right)$. When $Z_{k \Delta t}$ belongs to an interface layer, we use a SNOB step around the interface (in which the other membrane is not "seen"). An algorithm for this step is easily deduced from (6) (see [9, 10] for the precise algorithm). A SNOB step is similar to a Gaussian step except for a possible symmetric replacement of the particle around the membrane according to the value of the local time and the one of $\xi_{k, 1}$.

For $t \geq 0$, we write $\zeta_{k}(t)=X_{k \Delta t}+\sqrt{2 D_{0}}\left(B_{t+k \Delta t}-B_{k \Delta t}\right)$. Thus, $\Xi\left(X_{k \Delta t}, \beta_{k}, \xi_{k}\right)=\Theta\left(X_{k \Delta t}, \beta_{k}, \xi_{k}\right)$ on the following event

$$
\begin{aligned}
& E_{k}=\left\{\zeta_{k} \text { does not hit any membrane during }[0, \Delta t]\right\} \\
& \qquad\left\{\left\{\zeta_{k} \text { hit only the membrane at } \pm \alpha \text { during }[0, \Delta t] \text { and }\left|X_{k \Delta t} \mp \alpha\right| \leq \delta\right\} .\right.
\end{aligned}
$$

In other words, $\Xi\left(X_{k \Delta t}, \beta_{k}, \xi\right)$ and $\Theta\left(X_{k \Delta t}, \beta_{k}, \xi_{k}\right)$ may give different values if (I) the two membranes are hit during $[0, \Delta t]$ (multiple membranes hits) or (II) $X_{k \Delta t}$ is not in an interface layer yet $\zeta_{k}$ reaches one membrane during $[0, \Delta t]$. 


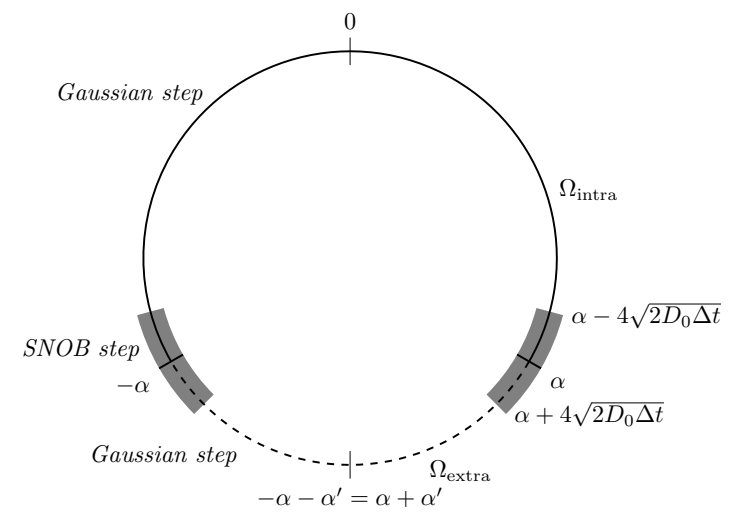

Figure 4: The periodic media and its interface layer (in gray).

We neglect the multiple membranes hits (I) since it implies that the range of $\left(\sqrt{2 D_{0}} B_{t}\right)_{t \in[0, \Delta t]}$ is larger than the distance between the membranes. We then focus on (II).

On the event $\cap_{i=0}^{n-1} E_{i}, Z_{n \Delta t}=X_{n \Delta t}$ and the simulation is exact. We now quantify the probability of such an event. If $\bar{A}$ denotes the complement of an event $A$,

$$
\mathbb{P}\left(\overline{\cap_{i=0}^{n-1} E_{i}}\right)=\mathbb{P}\left(\cup_{i=0}^{n-1} \overline{E_{i}}\right) \leq \sum_{i=0}^{n-1} \mathbb{P}\left(\overline{E_{i}}\right)
$$

Using the Markov property, we set $\zeta(t)=x+\sqrt{2 D_{0}} B_{t}$ for some point $x$ and we consider the events:

$$
\begin{aligned}
A(x) & =\left\{\zeta \text { hits }-\alpha \text { or } \alpha \text { during }[0, \Delta t] \text { with } \zeta_{0}=x \in \Omega_{\text {intra }}\right\} \\
A^{\prime}(x) & =\left\{\zeta \text { hits }-\alpha \text { or } \alpha \text { during }[0, \Delta t] \text { with } \zeta_{0}=x \in \Omega_{\text {extra }}\right\}
\end{aligned}
$$

The event $A(x)$ may then be decomposed as the (non disjoint) union of four events

$$
\begin{gathered}
A_{1}(x)=\left\{\zeta_{0}=x \in(-\alpha, \alpha), \zeta_{\Delta t} \notin(-\alpha, \alpha) \text { and } \alpha \text { has been hit }\right\} \\
A_{2}(x)=\left\{\zeta_{0}=x \in(-\alpha, \alpha), \zeta_{\Delta t} \notin(-\alpha, \alpha) \text { and }-\alpha \text { has been hit }\right\} \\
A_{3}(x)=\left\{\zeta_{0}=x \in(-\alpha, \alpha), X_{\Delta t} \in(-\alpha, \alpha) \text { and } \alpha \text { has been hit }\right\} \\
\text { and } A_{4}(x)=\left\{X_{0}=x \in(-\alpha, \alpha), X_{\Delta t} \in(-\alpha, \alpha) \text { and }-\alpha \text { has been hit }\right\} .
\end{gathered}
$$

A similar decomposition holds for $A^{\prime}(x)$. Using a symmetry argument, $A_{3}(x)=A_{4}(-x)$ and $A_{1}(x)=$ $A_{2}(-x)$.

The probability that a Brownian bridge hits a barrier is known, so that by conditioning over the value of $\zeta_{\Delta t}=y$, it holds that with $\Delta=2 D_{0} \Delta t$,

$$
\mathbb{P}\left(A_{4}(x)\right) \approx \int_{-\alpha+\delta}^{\alpha-\delta} \exp \left(\frac{-2(x-\alpha)(y-\alpha)}{\Delta}\right) \exp \left(\frac{-(y-x)^{2}}{2 \Delta}\right) \frac{\mathrm{d} y}{\sqrt{2 \pi \Delta}} .
$$

After some algebra, and neglecting multiple membranes hits,

$$
\mathbb{P}\left(A_{4}(x)\right)=\mathbb{P}\left(A_{3}(-x)\right) \approx \int_{-\alpha+\delta}^{\alpha-\delta} \exp \left(\frac{-(2 \alpha-x-y)^{2}}{2 \Delta}\right) \frac{\mathrm{d} y}{\sqrt{2 \pi \Delta}}=\Phi\left(\frac{-\alpha+x}{\sqrt{\Delta}}-\delta\right)-\Phi\left(\frac{-3 \alpha+x}{\sqrt{\Delta}}-\delta\right) .
$$

Again, by neglecting multiple membranes hits,

$$
\mathbb{P}\left(A_{2}(x)\right)=\mathbb{P}\left(A_{1}(-x)\right) \approx \Phi\left(\frac{-\alpha-x}{\sqrt{\Delta}}\right)-\Phi\left(\frac{-\alpha-2 \alpha^{\prime}-x}{\sqrt{\Delta}}\right) .
$$


The probabilities $\mathbb{P}\left(A_{i}^{\prime}(x)\right)$ are estimated the same way by exchanging $\alpha^{\prime}$ and $\alpha$.

Using (13), by neglecting multiple membranes hits during one time step,

$$
p=\mathbb{P}\left(\overline{\cap_{i=0}^{n-1} E_{i}}\right) \lesssim \sum_{k=0}^{n-1}\left(\mathbb{P}\left(A\left(X_{k \Delta t}\right)\right)+\mathbb{P}\left(A^{\prime}\left(X_{k \Delta t}\right)\right)\right)
$$

To simplify the computations, we assume that the initial distribution of the process follows the invariant measure, which is the Lebesgue's one. Hence, each $X_{k \Delta t}$ is uniformly distributed over $\Omega$. Thus, for $i=1, \ldots, 4$,

$$
\mathbb{P}\left(A_{i}\left(X_{k \Delta t}\right)\right)=\frac{1}{2 \alpha+2 \alpha^{\prime}} \int_{-\alpha+\delta}^{\alpha-\delta} A_{i}(x) \mathrm{d} x \text { and } \mathbb{P}\left(A_{i}^{\prime}\left(X_{k \Delta t}\right)\right)=\frac{1}{2 \alpha+2 \alpha^{\prime}} \int_{-\alpha^{\prime}+\delta}^{\alpha^{\prime}-\delta} A_{i}^{\prime}(x) \mathrm{d} x .
$$

For a numerical result, we use the following parameters taken from Table 1 ; $\alpha=V_{\text {intra }} / 2=10 \mu \mathrm{m}$, $\alpha^{\prime}=V_{\text {extra }} / 2=1 \mu \mathrm{m}$ and $D_{0}=3 \times 10^{-3} \mu \mathrm{m}^{2} \mu \mathrm{s}^{-1}$ for a simulation up to $T=5 \times 10^{4} \mu \mathrm{s}$. This choice that maximizes the number of times a Gaussian step is performed. The time step is $\Delta t=1 \mu$ s so that $5 \times 10^{4}$ steps are performed.

Thus, $p \lesssim 5 \times 10^{4} \times 2.2 \times 10^{-6}=0.11$. This implies that for more than $89 \%$ of the particles, the exact and the approximate schemes lead to the same sequence of positions. The actual value is greater than this because we have only estimated the number of time the particle hit one of the membranes when it starts away from the interface layer. However, this does necessarily not lead to a bad replacement. A finer analysis could then be performed using the distribution of the local time of a Brownian bridge.

Acknowledgement. The author wishes to thank J.-R. Li (Inria) who have proposed this research subject and provided the data of Table 11. This work has been supported by the ANR projects SIMUDMRI (ANR-10-COSI-0008) and H2MNO4 (ANR-12-MONU-0012-01).

The author also wishes to thank the referees for their careful reading that led to numerous improvements.

\section{References}

[1] A. Bobrowski. "Families of Operators Describing Diffusion Through Permeable Membranes". In: Operator Semigroups Meet Complex Analysis, Harmonic Analysis and Mathematical Physics. Ed. by W. Arendt, R. Chill, and Y. Tomilov. Springer International Publishing, 2015, pp. 87-105. DOI: $10.1007 / 978-3-319-18494-4 \_6$.

[2] M. Bossy, N. Champagnat, S. Maire, and D. Talay. "Probabilistic interpretation and random walk on spheres algorithms for the Poisson-Boltzmann equation in molecular dynamics". In: M2AN Math. Model. Numer. Anal. 44.5 (2010), pp. 997-1048. DOI: 10.1051/m2an/2010050.

[3] J. Coatléven, H. Haddar, and J.-R. Li. "A macroscopic model including membrane exchange for diffusion MRI”. In: SIAM J. Appl. Math. 74.2 (2014), pp. 516-546. DOI: 10.1137/130914255.

[4] E. Fieremans, D. S. Novikov, J. H. Jensen, and J. A. Helpern. "Monte Carlo study of a twocompartment exchange model of diffusion". In: NMR in Biomedicine 23.7 (2010), pp. 711 724.

[5] D. Gilbarg and N. S. Trudinger. Elliptic partial differential equations of second order. Classics in Mathematics. Springer-Verlag, Berlin, 2001.

[6] J. Kärger, H. Pfeifer, and W. Heink. "Principles and applications of self-diffusion measurements by nuclear magnetic resonance". In: Advances in Magnetic Resonance. Ed. by J. S. Waugh. Vol. 12. Academic Press, Inc., 1988, pp. 1-89. 
[7] O. A. Ladyženskaja, V. J. Rivkind, and N. N. Ural/ceva. "Classical solvability of diffraction problems for equations of elliptic and parabolic types". In: Dokl. Akad. Nauk SSSR 158 (1964), pp. 513-515.

[8] O. A. Ladyženskaja, V. A. Solonnikov, and N. N. Ural'ceva. Linear and quasilinear equations of parabolic type. Vol. 23. Translations of Mathematical Monographs. American Mathematical Society, Providence, R.I., 1968.

[9] A. Lejay. Estimation of the mean residence time in cells surrounded by semi-permeable membranes by a Monte Carlo method. Research report RR-8709. Inria, 2015.

[10] A. Lejay. “The snapping out Brownian motion”. In: Ann. Appl. Probab. 26.3 (2016), pp. 17721742. DOI: 10.1214/15-AAP1131.

[11] A. Lejay. "Simulation of a stochastic process in a discontinuous layered medium". In: Electron. Commun. Probab. 16 (2011), pp. 764-774. DOI: 10.1214/ECP.v16-1686.

[12] A. Lejay and S. Maire. "Computing the principal eigenvalue of the Laplace operator by a stochastic method". In: Math. Comput. Simulation 73.6 (2007), pp. 351-363. DOI: $10.1016 /$ j matcom.2006.06.011.

[13] A. Lejay and G. Pichot. "Simulating diffusion processes in discontinuous media: a numerical scheme with constant time steps". In: J. Comput. Phys. 231.21 (2012), pp. 7299-7314. DOI: $10.1016 / j \cdot j c p .2012 .07 .011$.

[14] D. Lépingle. "Euler scheme for reflected stochastic differential equations". In: Math. Comput. Simulation 38.1-3 (1995). Probabilités numériques (Paris, 1992), pp. 119-126. DOI: 10.1016/ 0378-4754(93)E0074-F.

[15] K. J. McGuire and J. J. McDonnell. "A review and evaluation of catchment transit time modeling”. In: Journal of Hydrology 330.3-4 (Nov. 2006), pp. 543-563.

[16] H. T. Nguyen, D. Grebenkov, D. V. Nguyen, C. Poupon, D. Le Bihan, and J.-R. Li. "Parameter estimation using macroscopic diffusion MRI signal models". In: Physics in Medicine and Biology 60.8 (2015), p. 3389. DOI: 10.1088/0031-9155/60/8/3389.

[17] M. Nilsson, D. van Westen, F. Ståhlberg, P. C. Sundgren, and J. Lätt. "The role of tissue microstructure and water exchange in biophysical modelling of diffusion in white matter". In: Magnetic Resonance Materials in Physics, Biology and Medicine 26.4 (2013), pp. 345-370. DOI: $10.1007 / \mathrm{s} 10334-013-0371-\mathrm{x}$

[18] Y. Pawitan. In all likelihood. Oxford University Press, 2013.

[19] R. Perrussel and C. Poignard. "Asymptotic expansion of steady-state potential in a high contrast medium with a thin resistive layer". In: Appl. Math. Comput. 221 (2013), pp. 48-65. DOI: $10.1016 /$ j.amc.2013.06.047.

[20] G. Pontrelli and F. de Monte. "Mass diffusion through two-layer porous media: an application to the drug-eluting stent". In: International Journal of Heat and Mass Transfer 50.17-18 (2007), pp. 3658-3669. DOI: 10.1016/j.ijheatmasstransfer.2006.11.003.

[21] D. Regan and P. Kuchel. "Mean residence time of molecules diffusing in a cell bounded by a semi-permeable membrane: Monte Carlo simulations and an expression relating membrane transition probability to permeability”. In: Eur. Biophys. J. 29.3 (2000), pp. 221-7.

[22] E. Sánchez-Palencia. Nonhomogeneous media and vibration theory. Vol. 127. Lecture Notes in Physics. Springer-Verlag, Berlin-New York, 1980.

[23] D. W. Stroock. "Diffusion semigroups corresponding to uniformly elliptic divergence form operators". In: Séminaire de Probabilités, XXII. Vol. 1321. Lecture Notes in Math. Springer, Berlin, 1988, pp. 316-347. DOI: 10.1007/BFb0084145. 
[24] J. R. S. Ureña, S. Olmos, and V. G. Kiselev. "Tissue-blood exchange of extravascular longitudinal magnetization with account of intracompartmental diffusion”. In: Magnetic Resonance in Medicine 66.5 (Apr. 2011), pp. 1445-1455.

[25] P. Veng-Pedersen. "Mean time parameters in pharmacokinetics. Definition Computation and Clinical Implications (Part I)". In: Clinical pharmacokinetics 17.5 (1989), pp. 345-366. DOI: 10.2165/00003088-198917050-00004.

[26] E. Zeidler. Nonlinear functional analysis and its applications. II/A. Linear monotone operators, Translated from the German by the author and Leo F. Boron. Springer-Verlag, New York, 1990. DOI: $10.1007 / 978-1-4612-0985-0$. 